\title{
DRAG OF SHOCK-ACCELERATED MICROPARTICLES
}

\author{
KATHY PRESTRIDGE \\ Los Alamos National Laboratory, USA
}

\begin{abstract}
Experiments of shock-accelerated microparticles show high drag coefficients when the particles are tracked from initial acceleration through the relaxation times. An eight-pulse particle tracking diagnostic measures individual particle positions, and a schlieren system measures shock location, with pressure transducers providing shock speed at the test section. These diagnostics give us detailed measurements of particle positions versus time for Mach 1.2, 1.3 and 1.4 experiments, allowing us to calculate accelerations and drag. Findings show that early-time drag is underestimated by current quasisteady drag models due to several factors. The exact physical mechanisms causing the large drag are likely due to body pressure forces caused by the particle wake disturbing the carrier phase.

Keywords: unsteady drag, microparticles, shocks, shock tube.
\end{abstract}

\section{INTRODUCTION}

The passage of a shock wave past microparticles causes them to accelerate behind the shock wave then relax into mechanical and thermal equilibrium with the post-shock gas [1]. Experimental studies of accelerated particles have been performed, but usually the experiments and drag models are limited to the acoustic time scale of the flow, when the shock is just passing across the particle [2]. The total forces on a rigid sphere are generally modelled using quasi-steady, inviscid unsteady, viscous unsteady, gravity, and lift forces [3]. Theoretical and empirical unsteady drag models are developed using linearized asymptotic analyses [4]-[6] for small oscillations about a mean flow around a sphere or empirical corrections [7]-[9] such as adding compressibility effects to the linearized BassetBoussinesq-Oseen (BBO) equation in the limit of zero particle Mach and Reynolds numbers. For shock-particle dynamics, these models do very well predicting drag on acoustic time scales, just when the shock is interacting with the particle. Unfortunately, these linearized models have limited applicability to real flows, where the particle Mach and Reynolds numbers are non-zero.

High drag coefficients have been observed by some experiments [10]-[14], but the experiments of unsteady drag behind a shock wave have mostly been limited to tracking large clouds of particles. High drag coefficients have been observed in several different experiments, but few have looked at individual particles to reduce uncertainty and study disperse flow conditions. Although high drag has been observed in post-shock flows, the physical mechanisms causing the high drag are suspected to be a time-dependent, nonlinear combination of inviscid drag forces. With these questions in mind, we have begun a series of experiments [15], [16] to observe microparticle behaviour in shock tubes and identify the physical mechanisms driving the high drag.

\section{EXPERIMENTAL FACILITY AND DIAGNOSTICS}

The experiments were performed at the Los Alamos Horizontal Shock Tube (HST) facility. The facility was designed to use a pneumatic, membraneless driver to produce shocks of $\mathrm{Ma}<2$. The set up for these experiments is described in [15], and the HST is pictured in Fig. 1. The cross-section of the HST is $76 \times 76 \mathrm{~mm}$ and the pneumatic driver is described in [17]. 


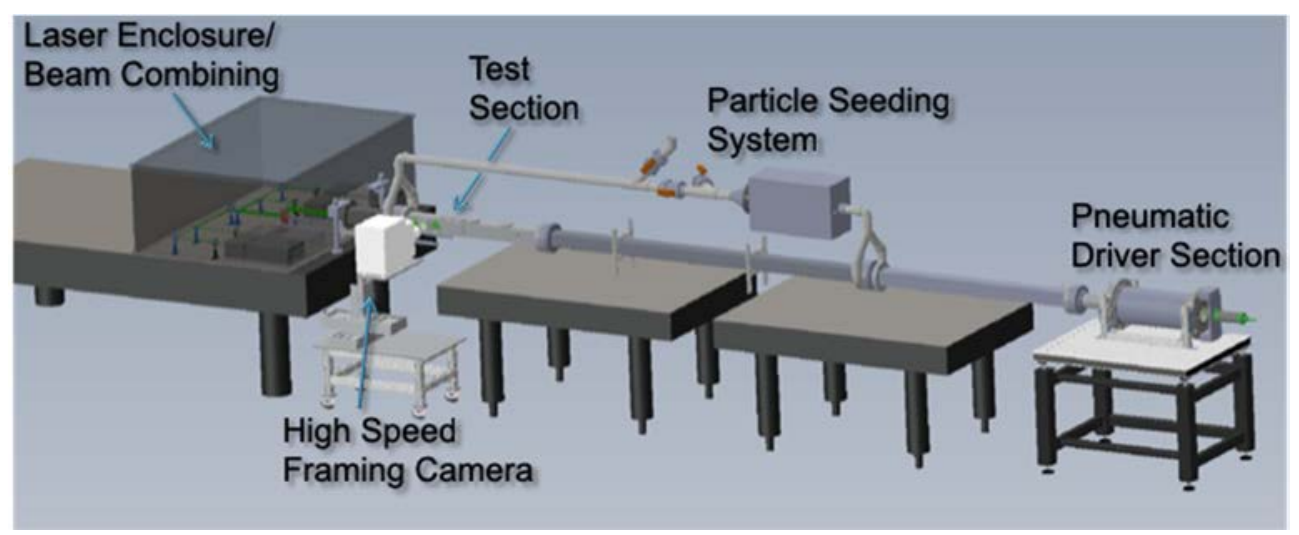

Figure 1: Horizontal shock tube with 8-pulse laser sheet system, high speed camera, and driver. The tube is $6.5 \mathrm{~m}$ long. The schlieren optics, not shown, are directed into the high speed framing camera from the opposite side of the test section. Pressure transducers run along the length of the driven section of the HST, all the way to the test section.

Experiments are performed using nylon particles (TSI 10084) with mean diameter of $4 \mu \mathrm{m}$ and density of $1140 \mathrm{~kg} / \mathrm{m}^{3}$. Particles are illuminated by four dual-head Nd:YAG 532 $\mathrm{nm}$ lasers, and each of the eight pulses is timed independently. The exact timing of the laser pulses is confirmed using a high speed photodetector. To resolve the small particles, and to ensure that the centroids of the particles can be determined in each image, significant magnification of the flow is necessary. The field of view of the framing camera is $2.74 \times 2.05 \mathrm{~mm}$, with a resolution of $2.14 \mu \mathrm{m} /$ pixel.

\section{EXPERIMENTAL RESULTS}

Fig. 2 shows measured particle positions for many different experiments, with four different particle tracks highlighted in colour. The particle drag, velocities and accelerations are calculated using a piecewise fitting method that is described in [18].

The position versus time measurements show that the particles are moving very quickly, and the calculated velocities and accelerations show that the early-time part of the flow, after the passage of the shock, is dominated by very high accelerations. This part of the flow is unsteady, gradually leading to particle relaxation to the post-shock flow speed at late times. Drag coefficients for the particles are calculated using the expression:

$$
\ddot{x}=\frac{3 \rho_{g}\left(u_{g}-\dot{x}_{p}\right)^{2}}{4 \rho_{p} d_{p}} C_{D}(t),
$$

where the dynamic properties are all post-shock. If $C_{D}(t)$ is known, this equation can be integrated numerically or analytically to determine an expression for the particle position versus time. The full description of the piecewise fitting method to determine drag coefficient from the particle position, including uncertainty analysis, can be found in Bordoloi et al. [18].

Particle drag is significantly higher than Clift-Gauvin, as shown in Fig. 3 for two different Mach number studies with the nylon particles. As the particles reach relaxation with the main flow, the drag gets closer to standard drag. Sets of experiments focusing on the initial 

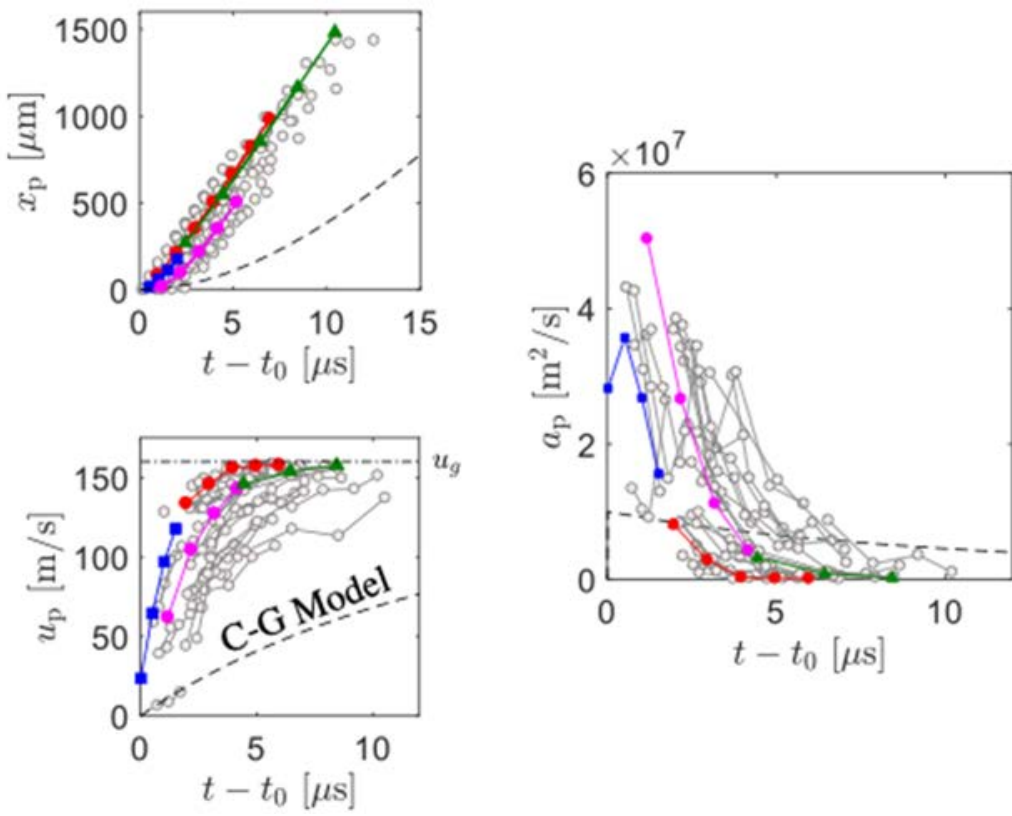

Figure 2: Particle positions, velocities and accelerations versus time after shock passage show much higher accelerations compared to those predicted by the CliftGauvin [19] model.
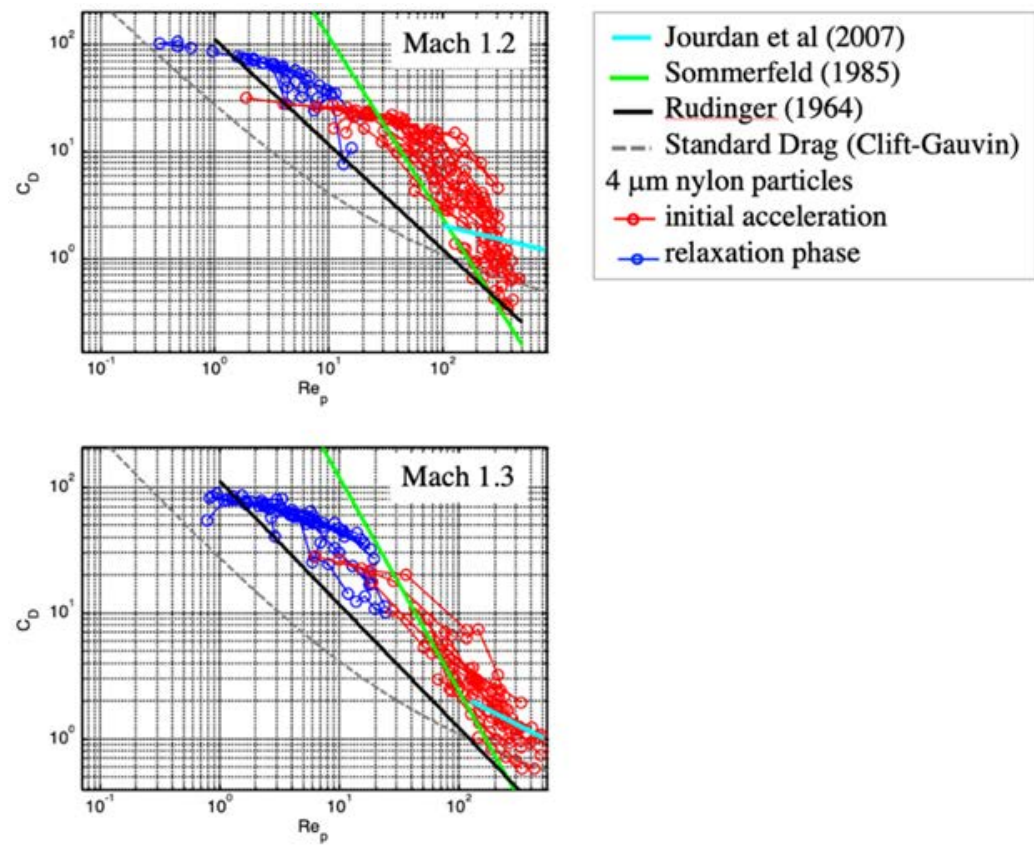

Figure 3: Drag coefficients from past and current shock-accelerated flow experiments. 
acceleration phase were performed (in red), but to capture the last part of the acceleration, experiments at later times had to be performed separately (in blue). This is because the limited field of view, for high resolution, did not allow the full range of particle acceleration, from rest to the freestream velocity, to be captured in one experiment. The latertime measurements (mostly in blue) are where the Reynolds number, defined as $R e_{p}(t)=d_{p}\left[u_{g}-u_{p}(t)\right] / v_{g}$ is approaching zero because the particle slip velocity $\left(u_{g}-u_{p}\right)$ is approaching zero, but there is no distinct separation between the early and late time experiments.

\section{ANALYSIS}

To better quantify drag as a function of the time scales of this unsteady flow, we look at the convective $\left(t^{*}\right)$ and acoustic $\left(\tau_{s}\right)$ time scales,

$$
t^{*}=d_{p} /\left|u_{g}-u_{p}(t)\right| \text { and } \tau_{s}=d_{p} / u_{s} .
$$

The convective time scale is dependent upon the particle slip velocity, that is decreasing with time. The ratio of the convective and acoustic time scales can be written as a modified Knudsen number for the flow, where $K n=M_{s} / R e_{p}$. The relationship between the Knudsen number and particle drag coefficient is shown in Fig. 4, where the drag coefficient increases sharply and becomes very high early on in the flow, and then it levels off.

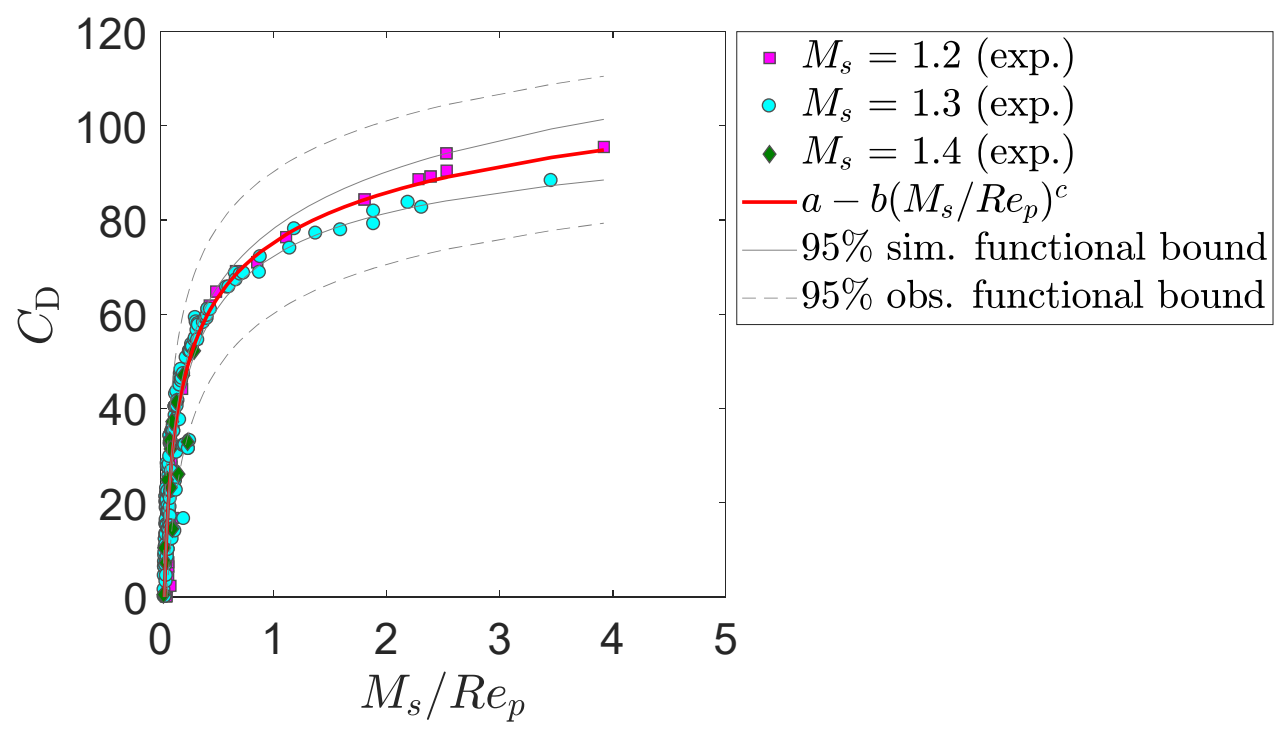

Figure 4: Drag coefficients as a function of the modified Knudsen number of the flow. The functional fit, in red, is $C_{\mathrm{D}}=167.5-92.2 \mathrm{Kn}^{-0.18}$.

This result is compared to the Clift-Gauvin [19] quasi-steady drag model in Bordoloi et al. [16], and the quasi-steady drag model only accounts for increased drag during the acoustic time scale. At the time of the shock passage, the experimental results agree with the unsteady drag models proposed by Parmar et al. [9], including: Clift-Gauvin (quasi-steady), Inviscid unsteady, and viscous unsteady (Bassett history). As soon as the particle starts accelerating, 
the experiments diverge from the model, and drag is dominated by an unsteady force for which there is no model currently.

The quasi-steady model of Clift-Gauvin is applicable over the full range of the experiment, since the constraints of $K n<<1$ and $M_{p}<0.6$ are met. For these experiments, $K n \approx 10^{-2}$ and $0.002<M_{p}<0.5$. The inviscid and viscous unsteady models proposed by Parmar et al. [9] have limited range of applicability. Namely, their inviscid unsteady model is applicable only when the times of the flow are smaller than the acoustic time scales, or $t<\tau_{s}$. In our experiments, $t \approx 1000 \tau_{s}$. Their viscous unsteady model is applicable only when the convective times of the flow are much smaller than the inverse of the product of the particle Reynolds number and shock Mach number, or $\tau_{c} R e_{p} M_{s} \ll 1$. In our experiments, $210.6<\tau_{c} R e_{p} M_{s}<436.8[16]$.

In unsteady flows, the classical Basset term is also not uniformly valid for long times, even at low particle Reynolds numbers. When a particle accelerates from rest, its approach to steady state scales as $t^{-2}$ instead of $t^{-1 / 2}$ [20].

We are left with a large unsteady force that is causing the particle to have very high drag. This means that the particle will accelerate much faster than the quasi-steady model predicts over times greater than the acoustic time scale, and this has implications for simulations of shock-driven multiphase flows.

Given the current limitations of unsteady drag models, we must rely on calculations to help us understand the forces on the particle. Current work at Los Alamos is to run simulations of a single particle that can move freely after shock passage. A possible reason for past simulations not seeing this high drag over long time scales is that in most cases, simulations hold the particle stationary. So, the conditions that the particle sees are quiescent flow and then a uniform constant velocity.

Fig. 5 shows a possible explanation for the experimental results. Initially, the particle is stationary in a quiescent flow (Initial Condition). A Mach 1.5, for example, shock then passes over the particle (Acoustic Time Scale). At that time, the particle has not begun to move, and

Initial Condition

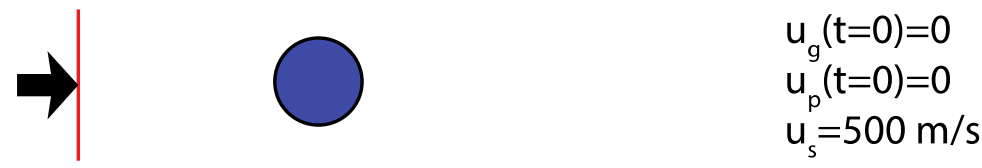

Acoustic Time Scale

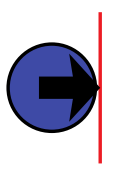

$u_{g}(t>0)=200 \mathrm{~m} / \mathrm{s}$

$u_{p}(t)=0$

$u_{s}=500 \mathrm{~m} / \mathrm{s}$

Relaxation Time Scale

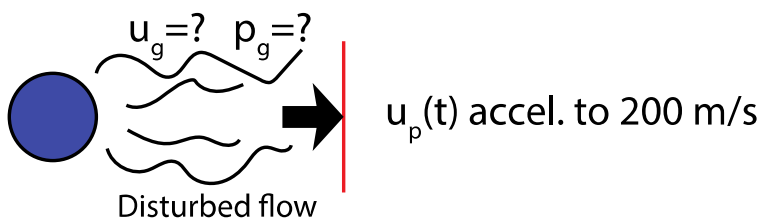

Figure 5: Possible particle dynamics affecting drag through relaxation times in shockdriven flows. 
the gas behind the shock is moving at about $200 \mathrm{~m} / \mathrm{s}$. The shock then moves away from the particle. This sets up a condition where the particle is starting to move, but it is moving into a disturbed flow, not a quiescent or constant velocity flow. The disturbance is caused by the wake of the particle after the shock passes. This will create lower pressure conditions, and the particle is getting accelerated into those conditions. Because of the impulsive force on the particle, it will not convect with its wake, but rather get accelerated into the wake. It is possible that this imbalance causes the very high initial accelerations seen in the experiments.

To better understand the mechanisms driving these microparticles, calculations are beginning at Los Alamos that will allow the particle to move freely in the flow field. Additionally, experiments are continuing that will examine drag on liquid droplets. We are also working on diagnostic improvements to measure the carrier phase velocities and potentially visualize the particle wake dynamics using molecular tagging velocimetry.

\section{ACKNOWLEDGEMENTS}

The experiments were carefully performed and analyzed by Greg Orlicz and Adam Martinez.

\section{REFERENCES}

[1] Rudinger, G., Some properties of shock relaxation in gas flows carrying small particles. Physics of Fluids, 7(5), pp. 658-663, 1964.

[2] Sun, M., Saito, T., Takayama, K. \& Tanno, H., Unsteady drag on a sphere by shock wave loading. Shock Waves, 14(1), pp. 3-9, 2005.

[3] Maxey, M.R. \& Riley, J.J., Equation of motion for a small rigid sphere in a nonuniform flow. Physics of Fluids, 1983.

[4] Miles, J.W., On virtual mass and transient motion in subsonic compressible flow. $Q$. J. Mech. Appl. Math., 4, pp. 388-400, 1951.

[5] Longhorn, A.L., The unsteady, subsonic motion of a sphere in a compressible inviscid fluid. Q. J. Mech. Appl. Math., 5, pp. 64-81, 1952.

[6] Saito, T., Saba, M., Sun, M. \& Takayama, K., The effect of an unsteady drag force on the structure of a non-equilibrium region behind a shock wave in a gas-particle mixture. Shock Waves, 17, pp. 255-262, 2007.

[7] Henderson, C., Drag coefficients of spheres in continuum and rarefied flows. AIAA Journal, 14(6), pp. 707-708, 1976.

[8] Loth, E., Compressibility and rarefaction effects on drag of a spherical particle. AIAA Journal, 46(9), pp. 2219-2228, 2008.

[9] Parmar, M., Haselbacher, A. \& Balachandar, S., Improved drag correlation for spheres and application to shock-tube experiments. AIAA Journal, 48, pp. 1273-1276, 2010.

[10] Rudinger, G., Some properties of shock relaxation in gas flows carrying small particles. Physics of Fluids, 7(5), pp. 658-663, 1964.

[11] Sommerfeld, M., The unsteadiness of shock waves propagating through gas particle mixtures. Exp. Fluids, 3, pp. 197-206, 1985.

[12] Igra, O. \& Takayama, K., Shock tube study of the drag coefficient of a sphere in a nonstationary flow. Proc. R. Soc. A, 442, pp. 231-247, 1993.

[13] Jourdan, G., Houas, L., Igra, O., Estivalezes, J.L., Devals, C. \& Meshkov, E.E., Drag coefficient of a sphere in a non-stationary flow: new results. Proc. R. Soc., 463, pp. 3323-3345, 2007.

[14] Sun, M., Saito, T., Takayama, K. \& Tanno, H., Unsteady drag on a sphere by shock wave loading. Shock Waves, 14(1), pp. 3-9, 2005. 
[15] Martinez, A.A., Orlicz, G.C. \& Prestridge, K.P., A new experiment to measure shocked particle drag using multi-pulse particle image velocimetry and particle tracking. Exp. Fluids, 56, p. 1854, 2015.

[16] Bordoloi, A., Martinez, A.A. \& Prestridge, K., Relaxation drag history of shock accelerated microparticles. J. Fluid Mech., 823, R4, 2017.

[17] Mejia-Alvarez, R., Wilson, B.M., Leftwich, M.C., Martinez, A.A. \& Prestridge, K.P., Design of a fast diaphragmless shock tube driver. Shock Waves, 25(6), pp. 635-650, 2015.

[18] Bordoloi, A., Ding, L., Martinez, A.A., Prestridge, K. \& Adrian, R.J., A new method to calculate unsteady particle kinematics and drag coefficient in a subsonic post-shock flow. Meas. Sci. Tech., 29(7), 074001, 2018.

[19] Clift, R. \& Gauvin, W.H., The motion of particles in turbulent gas streams. Proc. Chemeca, 70(1), pp. 14-28, 1970.

[20] Mei, R. \& Adrian, R.J., Flow past a sphere with an oscillation in the free-stream velocity and unsteady drag at finite Reynolds number. J. Fluid Mech., 237, 1992. 